\title{
A REVIEW ON EXPERIMENTAL STUDIES OF THE PHOTOABSORPTION SPECTRA OF LOW $z$ IONS
}

\author{
P. NICOLOSI*, E. JANNITTI and G. TONDELLO* \\ Istituto Gas Ionizzati, CNR, Via Gradenigo 6/A, I-35131 Padova, Italy \\ * Dipartimento di Elettronica e Informatica, Via Gradenigo 6/A, I-35131 \\ Padova, Italy
}

\begin{abstract}
The experimental technique for obtaining absorption spectra of ionized species by using two laser produced plasmas and the experimental results on photoabsorption spectra of low $Z$ ions: Be, B, $C$ are reviewed. Measurements of photoionization cross-sections have been derived by extrapolating to the continuum the known oscillator strengths of discrete lines.
\end{abstract}

\section{1 - INTRODUCTION}

The technique of two laser produced plasmas (LPP) for obtaining absorption spectra of moderately charged ions has been widely applied both in the normal and in the grazing incidence spectral regions. The two LPP's are used respectively as a continuum radiation source and as an absorbing medium.

The LPP has been proved to be an intense source of XUV-VUV radiation, the characteristic of the emission depending on the target material and on the laser power density on the target $/ 1 /$. It results that the ablation region, with the highest electron density, emits a very strong and smooth continuum when suitably high $\mathrm{z}$ target materials are used. Typically the duration of the emission follows the time behaviour of the laser pulse. On the other hand the LPP of low Z solid materials seems to be the best absorbing medium in experiments on ionized species. Indeed there is no Iimitation in principle to the stage of ionization, only few joules of laser energy being enough to produce H-like ions with ionization potential of a few hundred eV. Furthermore the expansion properties of the LPP's show a good separation among different ionization stages. In fact the various ionized species have different time evolution and spatial distribution /2/. Firstly H-like and He-like ions are produced and have life times comparable to the laser pulse width, then the lowest ionization stages appear later, during the cooling phase of the target with a longer time duration. In addition the LPP expands in a cone-like shape around the normal to the target, where the highly charged ions are mostly concentrated close to the normal, while the lowest charged ones are distributed in a wider region mostly near the target surface. Actually the spatial distribution of the various ions as well as the ionization degree depend strongly on the laser irradiation of the target i.e, the focal spot size, the laser power density and distribution. By taking into account the expansion properties of the LPP's it is possible to resolve among the spectra of different ions.

With the present technique the spectra of Be I,II,III,IV have been observed between 40-110 $\dot{A}$ and the photoionization cross-sections of Be IV and III have been measured /3/. The spectra of B IV and III have been observed in the XUV region /4/, while those of $B$ II and Be II have been studied in the normal incidence $/ 5,6 /$. The photoabsorption cross-section of the transitions $2 s^{2}{ }^{1} s-2 s \epsilon P^{1} P$ and $2 s 2 p^{3} P-2 s \epsilon d^{3} D$ of $B$ II as well as of the transitions $2 s^{2} s-\epsilon d$ ${ }^{2} \mathrm{P}$ and $2 \mathrm{P}{ }^{2} \mathrm{P}-\epsilon \mathrm{d}{ }^{2} \mathrm{D}$ of $\mathrm{Be}$ II have been measured. 
Recently some experiments have been made on carbon ions in the range $25-45 \dot{A}$. Here we report on some earlier and more recent results, after a description of the experimental technique.

\section{2 - $\underline{\text { THE EXPERIMENT }}$}

The experimental set-up used in the grazing incidence region is shown in fig. 1. Two plasmas are generaici with a suitable time delay by focusing two laser beams on the targets $\mathrm{T}_{1}, \mathrm{~T}_{2}$.

The two beams have been split from a ruby laser beam of about $8 \mathrm{~J}$ of energy and 15-20 ns of duration. The beam 1 , carrying most of the laser energy, $>658$, is sharply focused onto targets of heavy elements ( $\mathrm{Cu}, \mathrm{Pb}, \mathrm{W}$ ) and produces a plasma emitting a strong and quite smooth continuous radiation in the soft $x$-ray range. The toroidal mirror $M$, working at grazing incidence, has largely different curvature radii: $\mathrm{R}=5600 \mathrm{~mm}$ in the meridional plane, i.e. the plane of the figure, and cross radius $r=26 \mathrm{~mm}$, and focuses the collected radiation firstly on the entrance slit of the spectrograph then at the diffracted wavelength position. For the particular arrangement the mirror $M$ looks directly at the ablation region on the target $T_{1}$. The laser beam 2 is focused with a spherocylindrical optical system on the target $\mathrm{T}_{2}$, close to the entrance slit of the spectrograph. $\mathrm{T}_{2}$ is of the proper element. The laser power density and the length of the focal spot on the target $\mathrm{T}_{2}$ can be optimized by using different beam splitters and by changing the focusing of the optical system; indeed also some slight defocusing can sometime be useful.

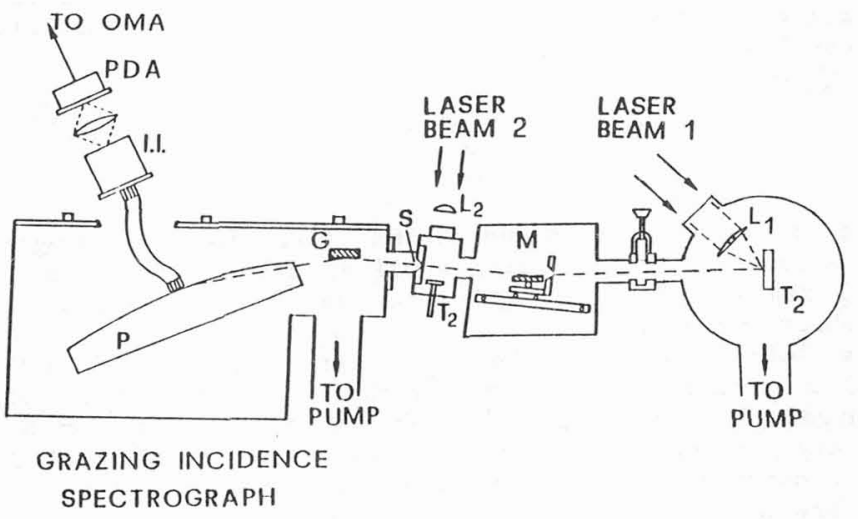

Fig. 1 - Experimental set-up used in the grazing incidence region.

In fig. 2 the experimental set-up used in the normal incidence region is shown. In this case the toroidal mirror $M$ has radii $R=640 \mathrm{~mm}$ in the meridional plane and cross radius $r=530$ $\mathrm{mm}$ and works at about $16^{\circ}$ of angle of incidence. The mirror focuses the radiation firstly at the position A corresponding to the Sirks focus of the spectrograph, where the absorbing plasma is created, then on the entrance slit $S$.

Since the absorbing plasma expands roughly in the direction normal to the target the adopted configurations minimize the effects of spatial gradients and Doppler effect on the. absorption process. In fact, also by suitably reducing the aperture of the toroidal mirxor, the absorbing plasma is irradiated by the background continuum in a very narrow region.

The absorbing plasma can be probed at different distances from the target, corresponding to various ion densities and ionization degrees, by moving the target itself. The delay between the absorbing plasma and the continuum emitting plasma can be varied too, in fact, as already mentioned, the various ionization stages have different time evolutions,

The detection system in the grazing incidence apparatus consists of a scintillator coated curved fiber optic face plate (FOFP) sliding along the Rowland circle. The FOFP is coupled, via a flexible fiber optic coherent bundle and a plane vacuum sealed FoFP, to an image intensifier (see fig. 1). The output signal is focused with high aperture objectives on a photodiode array of 512 pixels (2.5 mm height, $25 \mu \mathrm{m}$ width), which is connected to an optical multichannel analyzer OMA and to a PC computer. Scintillator coatings of TPB and TV type phosphors (P45) of thickness about $0.1-2 . \mathrm{mg} / \mathrm{cm}^{2}$ have been used. 
The whole instrumental function of the system has been measured, for example for a $6 \mu \mathrm{m}$ wide entrance slit, to be Lorentzian shaped with 4.5 pixels FWHM. However the optimum slit width for having the best results is a function of the spectral resolution and of the signal to noise $(\mathrm{S} / \mathrm{N})$ ratio. A carefull measurement of the instrumental function and a sufficiently high $S / N$ ratio coupled with a constrained deconvolution procedure have shown that results comparable to photographic detection techniques can be obtained $/ 3 /$.

In the normal incidence the detection system was consisting of a plate coated with $\approx 1$ $\mathrm{mg} / \mathrm{cm}^{2}$ TPB imaged via two high aperture objectives on an intensified photodiode array. connected with the OMA and a PC.

\section{3 - OBSERVATIONS}

Beryllium Spectra. In fig. 3 the absoxption coefficient for the Be spectra in the region between 110 and $45 \AA$ is reported. These spectra correspond to transitions of the 1 s electron

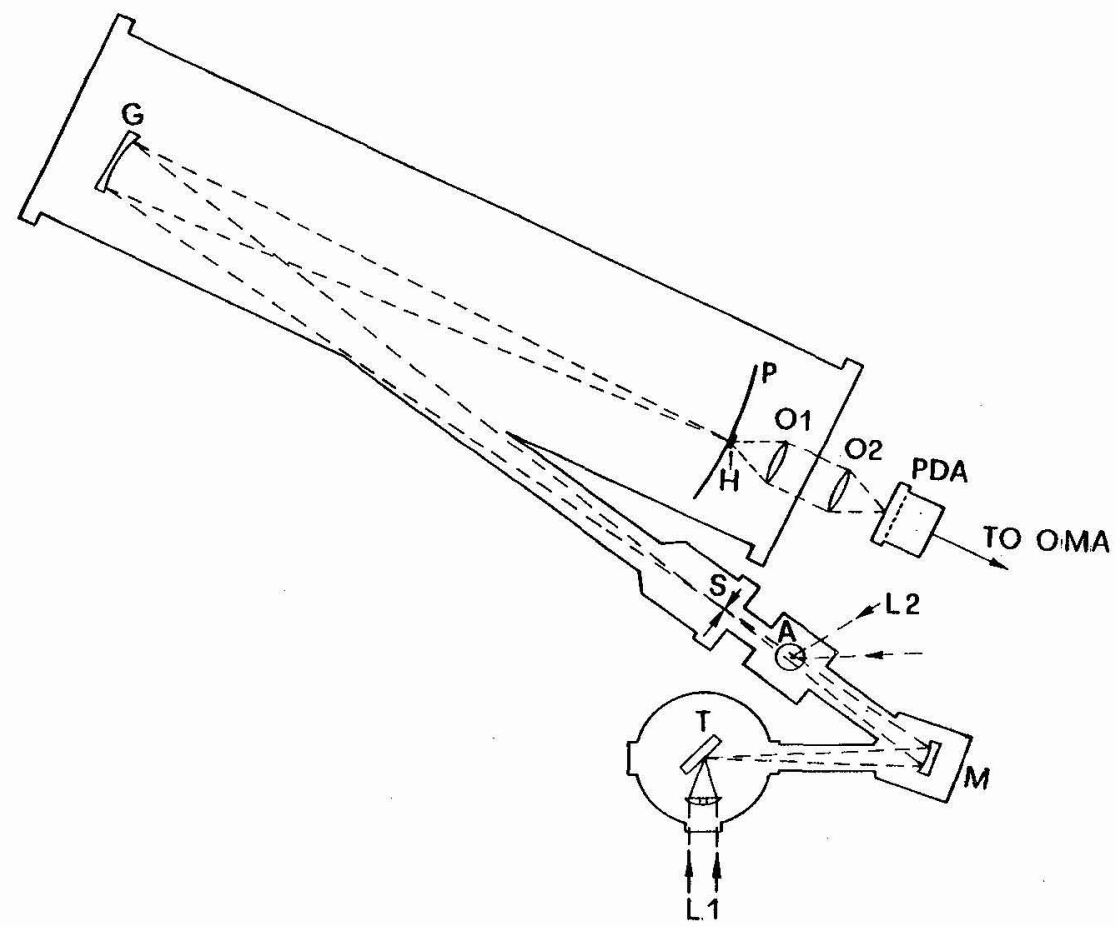

Fig. 2 - Experimental set-up used in the normal incidence region.

of the ions Be I,II,III, IV respectively from bottom to top. The high selectivity of the experimental technique among the various ionization stages is impressive.

The absorption coefficient is derived as

$$
k(\lambda)=\ln \left[I_{0}(\lambda) / I(\lambda)\right]
$$

where $I_{0}(\lambda)$ and $I(\lambda)$ are respectively the spectra recorded without and with the absorbing plasma. The recorded spectra have been corrected for the contributions due to stray light and to some emission from the absorbing plasma, the latter being mostly present when higher is the laser power density focused on the absorbing plasma target. The use of photodiode array with computer controlled data acquisition permits to derive easily the corrected value of the absorption coefficient.

The spectra show both discrete and continuous transitions with a clear jump corresponding to the photoionization limit. Due to the finite instrumental resolving power the discrete spectrum can be strongly altered; in order to derive the true absorption coefficient a deconvolution procedure has to be applied.

The instrumental function has been independently measured using very narrow line profiles 

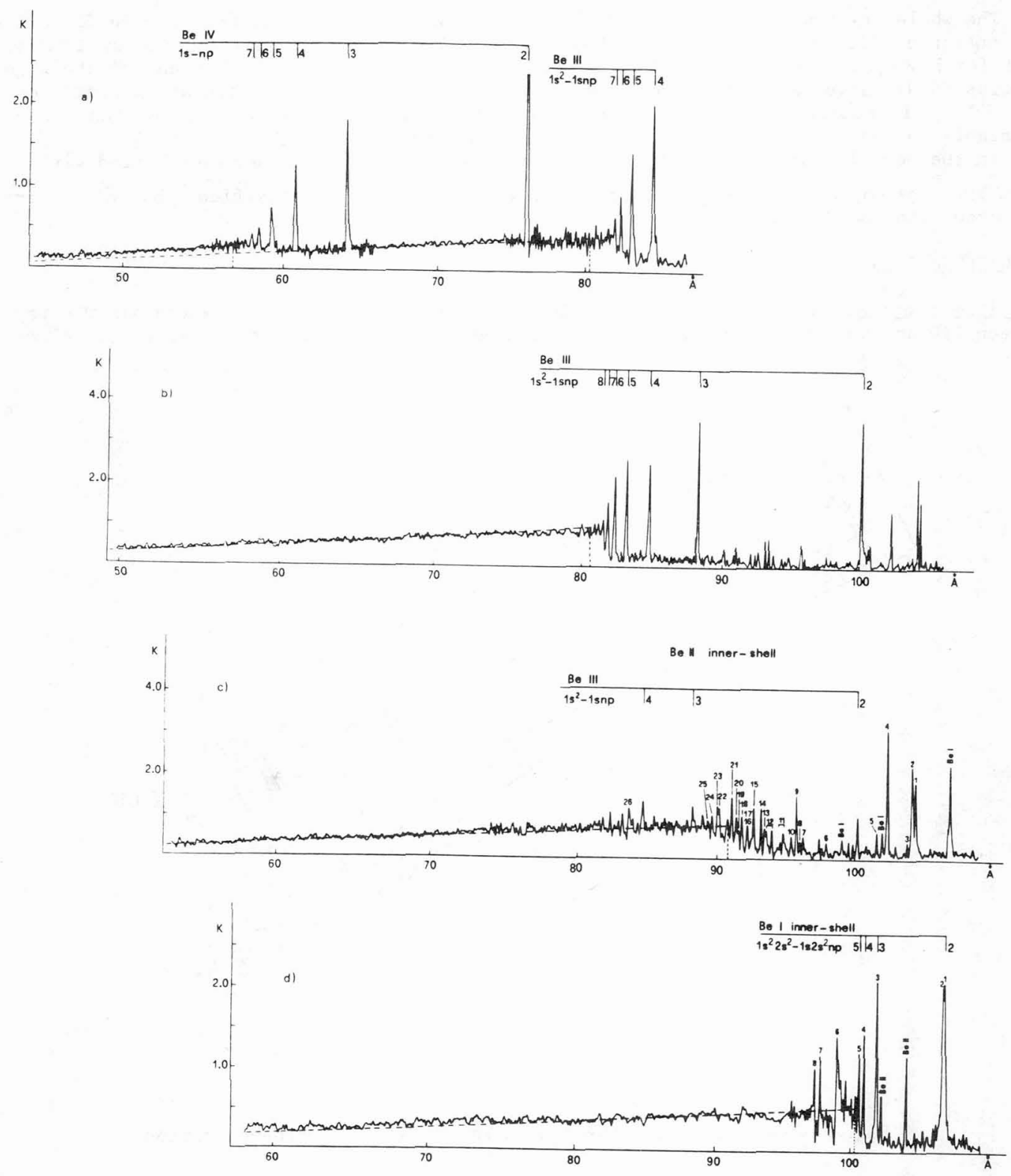

Fig. 3 - Absorption coefficient of a) Be IV, b) Be III, c) Be II, d) Be I.

both in emission and in absorption. Then a constrained deconvolution procedure has been applied to the absorptance, $A(\lambda)=1-I(\lambda) / I_{0}(\lambda)$, which is constrained between 0 and 1 . The iterative process used the Van Cittert algorithm with a non linear relaxation coefficient $13 /$. The deconvoluted data are then reconverted to absorption coefficient. The method has been successfully tested; it gives very good results if the instrumental profile is quite narrow with respect to the observed line width and the $\mathrm{S} / \mathrm{N}$ ratio is high enough. On the other hand by applying a selective multipoint smoothing routine prior and during the deconvolution process the method works very well even for $\mathrm{S} / \mathrm{N}$ ratios of 20 or 10 . The $\mathrm{S} / \mathrm{N}$ ratio depends strongly on the detected continuum radiation level and can be increased by cumulating several (10-30) exposures, provided a high shot by shot reproducibility and stability of the experiment.

In the figure the different noise level appearing in the various parts of the spectra is due 
to the different deconvolution process applied in the corresponding spectral intervals. The series due to resonant transitions in Be III and IV ions are very evident; the spectra of Be II and I are due to transitions of an inner electron and appear more complex. The Be II transitions are mostly of the type $1 s^{2} 2 s^{2} S-1 s 2 s n p{ }^{2} P$ and $1 s^{2} 2 p{ }^{2} P-1 s 2 p n p{ }^{2}$ (S,P,D) while those of $\mathrm{Be} I$ are of the $1 \mathrm{~s}^{2} 2 \mathrm{~s}^{2}{ }^{1} \mathrm{~s}-1 \mathrm{~s} 2 \mathrm{~s}^{2} \mathrm{np}{ }_{\mathrm{P}}$ configuration, in addition some $1 \mathrm{~s}^{2} 2 \mathrm{~s}^{2}$ $1_{S-1 s 2 s 2 p n s} 1_{P}$ lines appear beyond the ionization limit and show clearly autoionized profiles. The proper identification of the lines according to the numbers in the figure is reported in ref. $/ 3,8 /$. The photoionization continua are fitted (dotted curves)with the corresponding photoionization cross-sections calculated by Reilman and Manson /7/ normalized in the cases of $\mathrm{Be}$ II and Be I at the threshold values. The photoionization cross-sections at threshold have been measured for Be IV and III by deriving the line density from discrete

lines of known oscillator strenghts $/ 3 /$. is the absorption oscillator strength, $\phi(\lambda)$ is the normalized line profile and $\int$ nd $l$ is the line density, while for the continuum is given by $\mathrm{k}_{\mathrm{c}}(\lambda)=\sigma(\lambda) \int \mathrm{nd} \ell$, where $\sigma(\lambda)$ is the

photoionization cross-section. Also in this case the finite instrumental resolving power has to be taken into account. A simple best fit procedure of the observed discrete spectrum has been applied. A synthetic spectrum of Voigt profiles for the absorption coefficient is created, is converted to an absorption spectrum then is convoluted with the instrumental function and finally is reconverted to an absorption coefficient distribution and compared with the experimental one. When the best fit is reached the synthetic spectrum corresponds to the true absorption coefficient, i.e. what we had observed with infinite resolving power. Free parameters in the fitting process are the line density and the normalized line profile, which affect the peak values and the width of the synthetic absorption coefficient spectrum. The measured values $\sigma=(0.32 \pm 0.10) \mathrm{Mb}$ for $\mathrm{Be}$ IV and $\sigma=(1.4 \pm 0.2) \mathrm{Mb}$ for Be III compares very well with the theoretical ones. In tab. 1 the experimental parameters corresponding to the spectra shown in fig. 3 are reported. There ' $y$ ' is the distance from the target surface, while 'delay' is the time, after the generation, at which the absorbing plasma has been irradiated by the background continuum. Actually it does not take into account the time that the absorbing plasma spends to cover the distance $y$. The other parameters refer to the focusing of the laser beam on the absorbing plasma target. The large variation of the 'delay' and the laser power density has to be noted.

TAB. I

$\mathrm{Be} I \mathrm{Be}$ II Be III Be IV

$\begin{array}{lclll}\mathrm{y} \text { [mm] } & 0.5 & 0.5 & 0.75 & 0.25 \\ \text { delay [ns] } & 150 & 65 & 20 & 9.6 \\ \text { focal area [mm } & 1.8 & 1.8 & 1.5 & 0.8 \\ \text { focal spot length [mm] } & 18 & 18 & 15 & 1 \\ \text { power density [W cm }{ }^{-2} \text { ] } & 10^{9} & 3 \cdot 10^{9} & 3.7 \cdot 10^{9} & 10^{10}\end{array}$

The Be II absorption spectrum has been observed also in the normal incidence region /6/. The spectra corresponding to the transitions $1 s^{2} 2 s^{2} s-1 s^{2} n p^{2} P^{\circ}$ and $1 s^{2} 2 p^{2} P^{\circ}-1 s^{2} n d{ }^{2} \mathrm{D}$ have been observed and the relative photoionization cross-sections $\sigma$ have been measured with the best fit method previously described. The $2 \mathrm{~s}-\epsilon \mathrm{p}$ and the $2 \mathrm{~s}-\epsilon \mathrm{d}$ cross-sections at threshold result $\sigma=(1.5 \pm 0.1) \mathrm{Mb}$ and $\sigma=(2.5 \pm 0.5) \mathrm{Mb}$ respectively. The former is in excellent agreement with theoretical calculations, while some discrepancy, about $50 \%$, is with the latter $/ 9 /$. In this case the experimental data resulted strongly affected by the noise, but on the other hand the line profiles were broader than in the $2 \mathrm{~s}-\epsilon \mathrm{p}$ spectrum. For this reason the uncertainty has been estimated no larger than the $2 \mathrm{~s}-\epsilon \mathrm{p}$ case; however it could have been a little underestimated. With this in mind the agreement with the theoretical calculation can be considered quite good. 
Boron Spectra. The Boron ions have been observed both in the grazing incidence:B IV $1 s^{2}-1 s n p$ lines and B III inner-shell transitions $/ 4 /$, and in the normal incidence $B$ II/5/. In this latter case the spectrum of B II in the range 400-1700 $\AA$ has been investigated. Series of lines arising from the ground ${ }^{1} S$ and the ${ }^{3} \mathrm{P}^{\circ}$ metastable levels have been observed followed

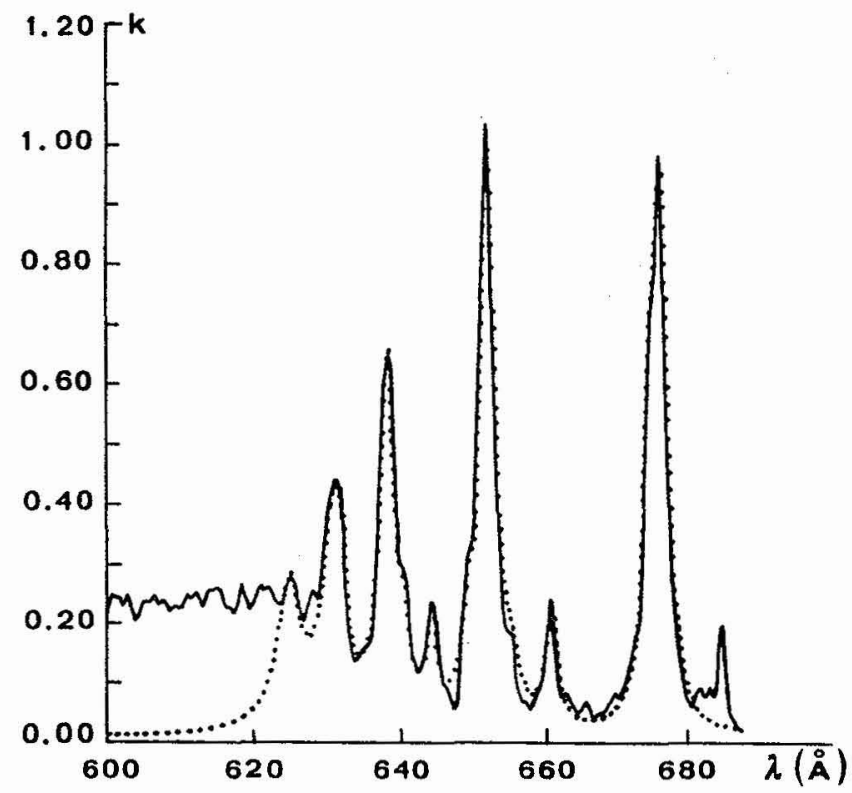

Fig. 4 - Absorption coefficient of the series $2 \mathrm{~s} 2 \mathrm{p}^{3} \mathrm{P}-2 \mathrm{snd}, \mathrm{ns}{ }^{3} \mathrm{D},{ }^{3} \mathrm{~S}$ of $\mathrm{B}$ II. Solid line: experiment; dotted line: best-fit model.

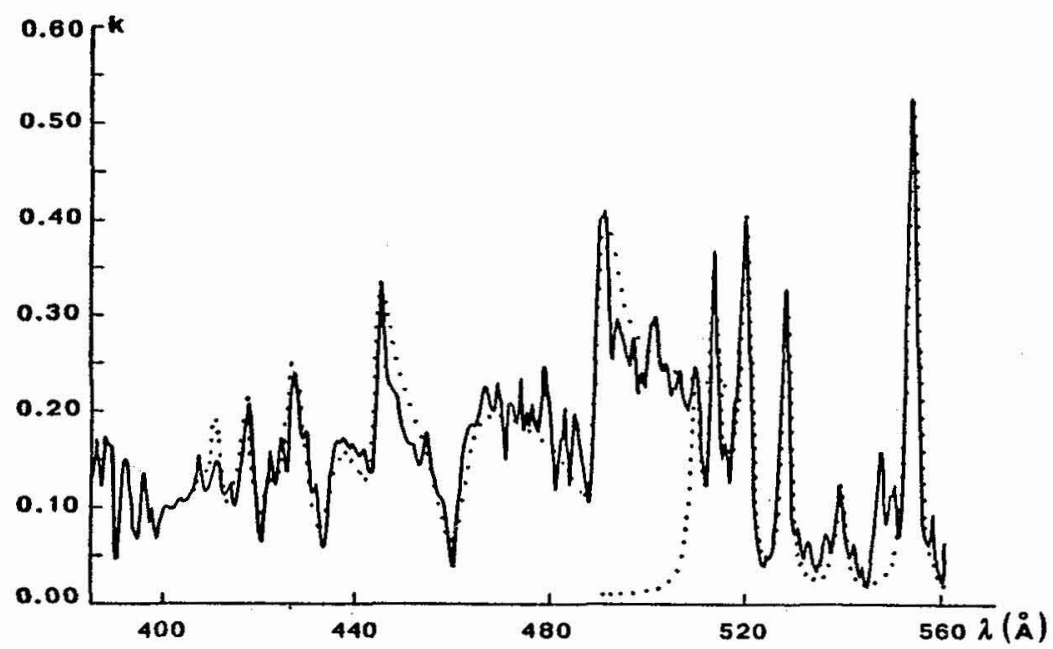

Fig. 5 - Absorption coefficient of the B II series $2 s^{2} 1_{S-2 p n d, n s} 1_{P}$ (left) and $2 s^{2} 1_{S-2 s n p}$ ${ }^{1} \mathrm{p}$ (right) - solid line: experiment, dotted line: best-fit model. 
by absorption continua. In fig. 4 the absorption coefficient for the series $2 s^{2} p^{3} \mathrm{P}-2 \mathrm{snd}$, ns ${ }^{3} \mathrm{D},{ }^{3} \mathrm{~s}$ while in fig. 5 that for the $2 \mathrm{~s}^{2}{ }^{1} \mathrm{~s}-2 \mathrm{snp}{ }^{1} \mathrm{P}$ and $2 \mathrm{~s}^{2}{ }^{1} \mathrm{~s}-2$ pnd,ns ${ }^{1} \mathrm{P}$ series are reported. The measured photoionization cross-section values at threshold are respectively for the $2 s^{2}-2 s \in p$ transition $\sigma=(1.7 \pm 0.6) \mathrm{Mb}$ and for the $2 \mathrm{~s} 2 \mathrm{p}-2 \mathrm{~s} \in \mathrm{d} \sigma=(4.0 \pm 0.5) \mathrm{Mb}$. In the $s h o r t$ wavelength part of the fig. 5 series of autoionized Iines identified as $2 s^{2}{ }^{1} \mathrm{~S}-2$ pnd,ns ${ }^{1} \mathrm{P}$ show characteristic Fano type profiles. The $2 s^{2}-2$ pns transitions show window type profiles while the $2 s^{2}-2$ pnd ones show asymmetric profiles. The corresponding spectrum has been fitted with a parametric formula according to Dubau and Seaton $/ 10 /$ with a simple modification for taking into account the interaction between the two series converging to the same limit /5/. Calculations of the photoionization cross-section of the $\mathrm{B}^{+}$have been reported by Tully et al. /11/. The cross-section has been calculated also between the first two ionization thresholds showing the cited autoionizing resonances. The authors make a detailed comparison of their results with the experimental ones, the agreement being excellent. In fig. 6 the calculated cross-section (full points) just above the threshold is reported and it has been fitted with the cited parametric formula. The open points with bars correspond to experimental data $/ 5 /$.

Carbon spectra. The carbon spectra are reported in figs. 7 and 8 . Fig. 7 shows the absorption coefficient of the ions C V, C IV and C III in the interval 25-45 $\dot{A}$. In table 2 . are reported the experimental parameters for these spectra. With the synthetic (Voigt profiles) deconvolution procedure the absorption coefficient, corrected for the instrumental distortion, and hence the line density of the $\mathrm{C} V$ ions has been measured /12/. The derived value for the photoionization cross-section at threshold is $\sigma=0.47 \mathrm{Mb}$ with a maximum

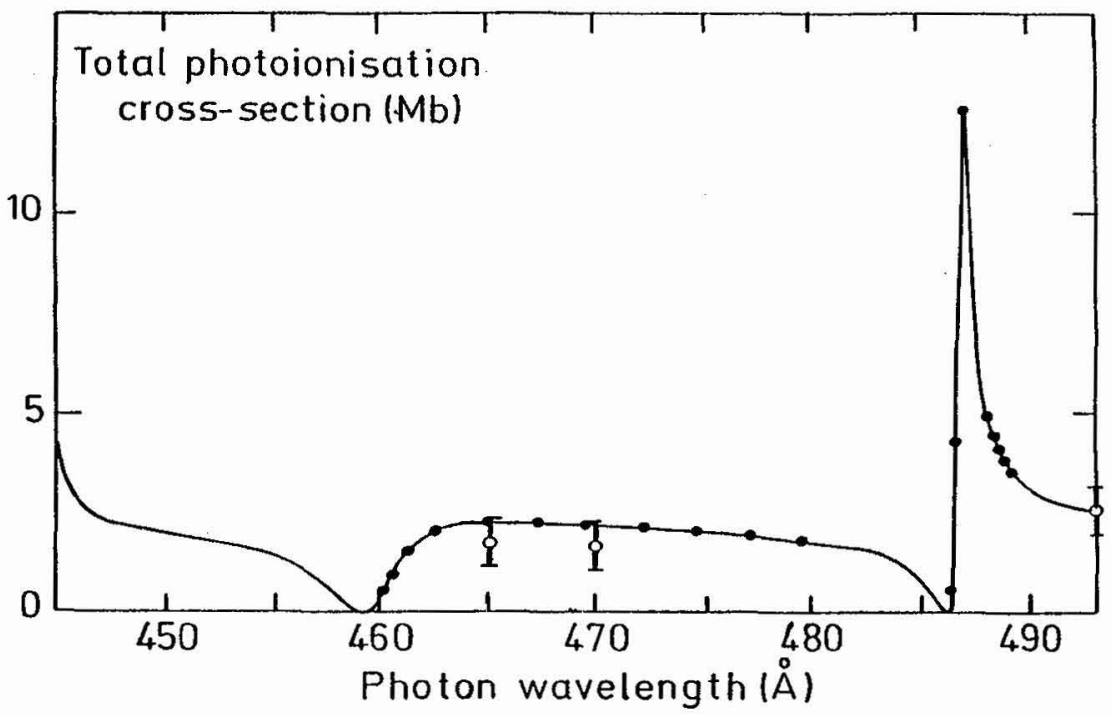

Fig. 6 - From ref. /11/. B II photoionization cross-section, see text and reference for details.

deviation of $0.05 \mathrm{Mb}$ over various independent measurements. The dotted lines correspond to the theoretical calculations /7/. The C V reported spectrum is an average between two independent observations with a slight smoothing process. The reduction of the contribution of the other ionization stages is noteworthy.

The C IV and C III spectra in the long wavelength part with discrete transitions have been deconvoluted with the constrained deconvolution procedure. Both spectra show discrete lines which for $C$ IV have been mostly identified as $1 s^{2} 2 s^{2} s-1 s 2 s n p{ }^{2} P$ and $1 s^{2} 2 p{ }^{2} p-1 s 2 p n p{ }^{2} P$ 


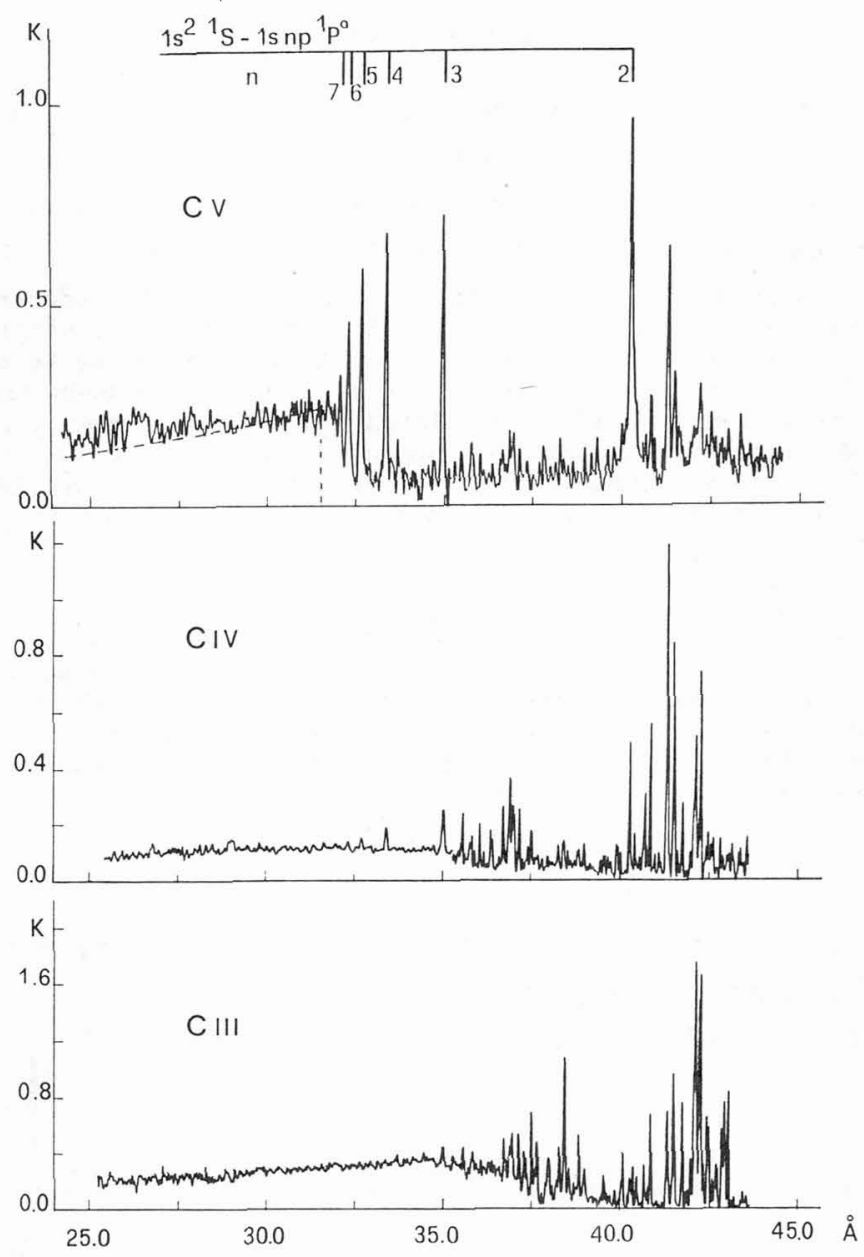

Fig. 7 - Absorption coefficient of 1s electron in carbon ions.

configurations, while for C III start from the ground level $1 s^{2} 2 s^{2}{ }^{1}$ and the low excited levels $1 \mathrm{~s}^{2} 2 \mathrm{~s} 2 \mathrm{p} \quad 1,3 \mathrm{P}$. A detailed analysis of these spectra is in progress. The photoionization continua are very clear and correspond mostly to $1 \mathrm{~s}-\epsilon \mathrm{p}$ transitions in both spectra. An absolute value of the photoionization cross-section has not been determined yet, although very interesting, because of the lack of measured transition probabilities for the. discrete inner-shell transitions. Furthermore in the C IV and C III spectra some lines are expected to be autoionized, however our present resolution is too low for observing the corresponding line shapes. The C IV and C III spectra show some mixing between ionization stages, i.e. C III lines are presented also in the C IV spectrum and viceversa. The main reasons are the range of abundance of Li-like and Be-like ions in an ionization balanced plasma, the duration of the background continuum emission ( $20 \mathrm{~ns})$ compared to the life time of Li-like and Be-like ions and the poor uniformity of the laser irradiation on the graphite target. In fig. 8 the spectra in the region 22-45 $\dot{A}$ of $C$ I carbon vapours and a solid graphite film are shown. Although the two LPP's method is mostly suited for the absorption spectroscopy of ions it appears also very interesting to extend its application to neutral atoms, vapours and solid state materials. In tab. II the experimental parameters for the $C$ spectra are reported. The focal spot sizes for the $\mathrm{CV}$ and $\mathrm{C}$ IV spectra were circular while these for the C III, C I, C vap were elongated. In fig. 8 the C I and vapour spectra have 


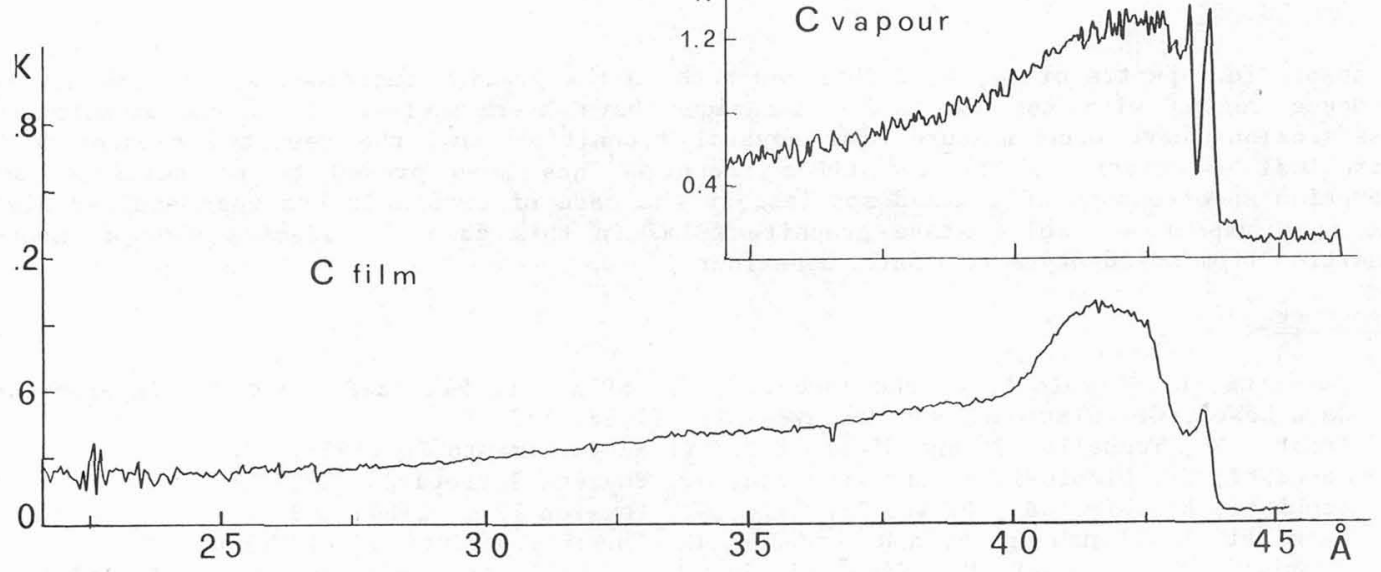

Fig. 8 - Absorption coefficient of neutral carbon.

been deconvoluted in the long wavelength portion. In the $C$ I spectrum the photoionization jump is clearly distinguishable around $41.5 \AA$ and the continuous spectrum extends down to 35 $\dot{A}$. It corresponds mostly to the photoionization of the 1 s electron from the lowest configurations $1 s^{2} 2 s^{2} 2 p^{2}{ }^{3} P,{ }_{D},{ }^{1} S$. The discrete transitions are mostly of the type $1 s^{2} 2 s^{2} 2 p^{2}-1 s 2 s^{2} 2 p^{3}$. The carbon vapour spectrum is obtained with considerably less power on the target and with the same 'delay' as for the C I spectrum, which is however quite longer than that used for obtaining the other carbon ions spectra. The spectrum looks like those corresponding to transitions from core levels to a valence state of solid or clusters compounds. There is not an immediate correspondence between the absorption peak in the $C$ vapour spectrum and the discrete lines of $C$ I. However it is likely that this peak can be interpreted as due to $1 s-2 p$ transition broadened and shifted by solid state levels. Finally

Table II

\begin{tabular}{|c|c|c|c|c|c|}
\hline & $\mathrm{c}_{\text {vap }}$ & C I & C III & C IV & $\mathrm{c} \mathrm{V}$ \\
\hline $\mathrm{y}[\mathrm{mm}]$ & 0.25 & 1.5 & 0.7 & 1.1 & 0.5 \\
\hline delay [ns] & 100 & 100 & 18 & 30 & 4.5 \\
\hline focal area $\left[\mathrm{mm}^{2}\right]$ & 42 & 4.9 & 4.8 & $\phi=1.1$ & $\phi=0.8$ \\
\hline focal spot length [mm] & 10 & 7 & 16 & 1.2 & 1 \\
\hline power density $\left[\mathrm{W} \mathrm{cm}^{-2}\right]$ & $1.1 \times 10^{7}$ & $7.0 \times 10^{8}$ & $2.0 \times 10^{9}$ & $4.0 \times 10^{9}$ & $10^{10}$ \\
\hline
\end{tabular}


as comparison the absorption spectrum from a $30 \mu \mathrm{g} / \mathrm{cm}^{2}$ thick amorphous graphite film is reported. Here the LPP has been used only as continuous radiation source. The shape of the absorption at shorter wavelengths appears different from the cluster and the $C I$ one and an absorption peak is present in the long wavelength part clearly corresponding to the peak observed in the vapour spectrum. It appears very well in agreement with the high resolution spectra taken by Del Grande and Tyrsell /13/ using synchrotron radiation. By considering the thickness of the target we derive a value for the photoabsorption coefficient of the graphite of $(1.85 \pm 30 \%) \mathrm{Mb}$ compared with the value of $(1.35 \pm 108) \mathrm{Mb}$ reported in ref. /13/. The. agreement, taking into account that in our case no particular effort has been made for a carefull determination of the thickness of the sample, is quite good.

\section{4 - CONCLUSIONS}

The absorption spectra of Be, B, C obtained both in the grazing incidence and in the normal incidence region with the two LPP's technique have been reviewed. The photoabsorption cross-sections have been measured for several transitions and the results compared with theoretical calculations. The two LPP's technique has been proved to be suitable for absorption spectroscopy of ionized species. In the case of carbon it has been applied also to C I, C vapour and solid state graphite film. In this case the spectra show a smooth transition from solid state to atomic behaviour.

\section{REFERENCES}

11/ Jannitti, E., Nicolosi, P. and Tondello, G., SPIE vol. 911 'X-Ray and VUV Interaction Data Bases, Calculations, and Measurements' (1988) 157.

12) Irrera, F., Tondello, G. and Nicolosi P., Il Nuovo Cimento 7D (1986) 626.

13/ Jannitti, E., Nicolosi, P. and Tonde11o, G., Physica Scripta 36 (1987) 93.

14/ Jannitti, E., Nicolosi, P. and Tondello, G., Physica 124C (1984) 139.

15/ Jannitti, E., Pinzhong, F. and Tonde1lo, G., Physica Scripta $\underline{33}$ (1986) 434.

16/ Jannitti, E., Mazzoni, M., Nicolosi, P., Tondello G. and Wang Yongchang, J. Opt.Soc., Am. $\underline{2 B}$ (1985) 1078 .

17/ Reilman, R.F. and Manson, S.T., Astrophys. J. Supp1. Ser40 (1979) 815.

18/ Jannitti, E., Nicolosi, P., Tondello, G., Zheng Yongzhen and Mazzoni, M., Opt. Commun. 63 (1987) 37 .

19/ Peach, G., Saraph, H.E. and Seaton, M.J., J. Phys. B 21 (1988) 3669.

/10/ Dubau, J. and Seaton, M.J., J. Phys. B 17 (1984) 381.

/11/ Tully, J.A., Le Dorneuf, M. and Zeippen, C.J., Astron. Astrophys 211 (1989) 485.

/12/ Jannitti, E., Nicolosi, P. and Tondello, G., Phys. Lett. A 131 (1988) 186.

13/ Del Grande, N.K. and Tirsell, K.G., SPIE vol. 911 'X ray and vUV Interaction Data Bases, Calculations and Measurements' (1988) 6. 